\title{
Clinical application: Restoration of immune homeostasis by autophagy as a potential therapeutic target in sepsis (Review)
}

\author{
LEMENG ZHANG $^{1}$, YUHANG AI ${ }^{1}$ and ALLAN TSUNG ${ }^{2}$ \\ ${ }^{1}$ Department of Intensive Care Unit, Xiangya Hospital, Central South University, Changsha, Hunan 410008, P.R. China; \\ ${ }^{2}$ Department of Surgery, University of Pittsburgh, Pittsburgh, PA 15213, USA
}

Received August 1, 2015; Accepted December 4, 2015

DOI: $10.3892 /$ etm.2016.3071

\begin{abstract}
Sepsis-induced lymphocyte and dendritic cell apoptosis contributes to immunosuppression, resulting in an inability to eradicate the primary infection and a propensity to acquire secondary infections. However, the inhibition of apoptosis may produce unexpected and undesirable consequences. Another cellular process, autophagy, is also activated in immune cells. There is increasing evidence to suggest that autophagy confers a protective effect in sepsis. The protective mechanisms underlying this effect include limiting apoptotic cell death and maintaining cellular homeostasis. Therefore, understanding the regulation of immune cell autophagy and apoptosis may provide insight into novel therapeutic strategies. The present review examined potential novel therapeutic strategies aimed at restoring immune homeostasis by inducing autophagy. The restoration of balance between apoptosis and autophagy may be a novel approach for improving sepsis-induced immunosuppression and decreasing susceptibility to sepsis.
\end{abstract}

\section{Contents}

1. Introduction

2. Immune cell apoptosis in sepsis

3. Immune cell autophagy in sepsis

4. Regulation immunity and inflammation

5. Reciprocal regulation between apoptosis and autophagy

6. Autophagy as a therapeutic target in pulmonary diseases

7. Autophagy as a potential therapeutic target for sepsis

8. Conclusion

Correspondence to: Dr Allan Tsung, Department of Surgery, University of Pittsburgh Medical Center, 3459 Fifth Avenue, Pittsburgh, PA 15213, USA

E-mail: tsunga@upmc.edu

Dr Yuhang Ai, Department of Intensive Care Unit, Xiangya Hospital, Central South University, 87 Xiangya Road, Changsha, Hunan 410008, P.R. China

E-mail: ayhicu1978@sina.com

Key words: autophagy, apoptosis, immunosuppression, sepsis

\section{Introduction}

Despite major advances in critical care management and antibiotic therapies, sepsis-induced multiple organ failure (MOF) continues to contribute to significant morbidity and mortality in intensive care units (ICUs) (1). MOF is the second leading cause of death among patients in non-coronary ICUs, and the tenth leading cause of death in the United States (2). In the United States, an estimated 250,000 individuals succumb to sepsis every year. The incidence of sepsis is increasing at a rate of $1.5 \%$ per annum and mortality rates of $\leq 70 \%$ have been estimated (3). Furthermore, this mortality rate has remained essentially unchanged for the past 25 years $(2,4)$.

Treating patients with severe sepsis and septic shock has been a great challenge. This is due to the incomplete understanding of the complex biological processes underlying sepsis, which has hindered the development of sepsis-specific therapies (1). Thus, it is essential to determine the mechanism underlying the pathophysiology of sepsis so that more effective therapeutic strategies may be developed.

Traditionally, sepsis has been considered to be an overwhelming inflammatory response, which results in MOF from which the patient ultimately succumbs (1). However, over the past 25 years, numerous clinical trials involving agents that block the inflammatory cascade, such as anti-endotoxin antibodies (5), interleukin-1 (IL-1) receptor antagonists (6), and tumor necrosis factor- $\alpha$ (TNF- $\alpha$ ) antagonists (7), have failed to improve the outcome of sepsis. The failure of anti-inflammatory agents highlights the fact that further research is required in order to elucidate the mechanisms underlying the septic response.

Numerous mechanisms underlie sepsis-induced immunosuppression, including apoptotic depletion of immune cells, increased $\mathrm{T}$ regulatory and myeloid-derived suppressor cells, and cellular exhaustion (8).

Recent studies demonstrated that sepsis perturbs immune homeostasis $(9,10)$. The pathophysiology of the septic response occurs in two distinct phases: A cytokine-mediated hyper-inflammatory phase, and a subsequent hypo-inflammatory and immunosuppressive phase $(11,12)$. During the later phase, the immune system exhibits defective antigen presentation, loss of delayed-type hypersensitivity response, loss of phagocytic function, decreased Th1 cytokine release, and increased apoptotic cell death of immune 
cells $(8,11)$. During this phase, patients fail to eradicate invading pathogens and are susceptible to opportunistic organisms. Most mortality cases occur during this phase (11). Notably, sepsis-induced immunosuppression occurs at a later stage, making it an attractive therapeutic target $(8,11)$.

Numerous interacting mechanisms underlying immunosuppression occur during sepsis, including increased T regulatory cells (13), increased myeloid-derived suppressor cells (14), apoptotic depletion of immune effector cells, and a shift from T helper cell (Th)1 to Th2 immune phenotype $(15,16)$. Several lines of evidences indicate that dysregulated lymphocyte and dendritic cell (DC) apoptosis are crucially involved in sepsis-induced immunosuppression $(1,11)$. Either direct apoptotic depletion of critical immune cells, such as B and $\mathrm{CD}^{+}$lymphocytes, or indirect apoptosis-induced anergy in surviving DCs may lead to host immunosuppression $(17,18)$. Strategies aimed at preventing immune cell apoptosis may be effective and improve survival in animal endotoxemia models $(12,19)$.

In addition to apoptosis, another cellular process, autophagy, has drawn increasing attention. Autophagy is activated in sepsis and exerts a protective role, by maintaining cellular homeostasis and limiting cell damage and death $(20,21)$. Previous studies have demonstrated that autophagy protects against sepsis-induced apoptotic cell death in the liver $(22,23)$ and kidney $(24,25)$, as well as lung injury $(26,27)$ and immune systems $(9,10)$.

Notably, increasing evidence suggests that crosstalk exists between apoptosis and autophagy. The 'choice' between autophagy and apoptosis may be integral to the fate of cells. The reciprocal regulation between apoptosis and autophagy may affect immune homeostasis in sepsis (28). The induction of autophagy may re-engage or preserve host immune homeostasis, and may avoid the side effects of complete inhibition of apoptosis (28).

The present review focused on the reciprocal regulation of apoptosis and autophagy, in order to provide insight into the molecular mechanisms that may be essential for the balance between immune cell survival and death. Therefore, autophagy may serve as a target for the development of novel therapeutic approaches.

\section{Immune cell apoptosis in sepsis}

Regulatory mechanisms and signaling pathways of apoptosis. Apoptosis is defined by characteristic changes as follows: Altered nuclear morphology including chromatin condensation and fragmentation, minor changes in cytoplasmic organelles, cell shrinkage, plasma membrane blebbing, and apoptotic body formation (29). At the molecular level, a complex termed the apoptosome, which consists of apoptosis protease-activating factor-1 (APAF-1), caspase-9, and cytochrome $c$, conducts apoptosis (30). Two major signaling pathways regulate the process of apoptosis: The intrinsic and the extrinsic signaling pathway.

In the intrinsic signaling pathway, stimuli lead to mitochondrial depolarization, release of cytochrome $c$, and activation of downstream caspase. Released cytochrome $c$ interacts with APAF-1 and pro-caspase-9 to form the apoptosome. This complex activates caspase-9, which then activates effector caspases (30). In the extrinsic signaling pathway, the initiating signal comes from the ligation of death receptors on the cell surface. Ligation of these receptors leads to the activation of initiator caspases in the cytoplasm and ultimately, converging with the intrinsic signaling pathway, and activation of downstream caspases and the apoptosome (30). Active caspase- 8 is able to directly activate effector caspases, such as caspase-3, or activate the intrinsic apoptosis signaling pathway by cleaving Bid, resulting in its translocation to the mitochondria (30). During sepsis, lymphocytes undergo extrinsic and intrinsic apoptosis (31).

Sepsis-induced immune cell apoptosis and immunosuppressive phase. As mentioned above, sepsis is characterized by an initial hyper-inflammatory response, followed by later immunosuppression $(11,12)$. Patients who succumb in ICUs following sepsis exhibit biochemical, flow cytometric, and immune histochemical results consistent with immunosuppression (32). Targeted immune enhancing therapy may be a valid approach in selected patients with sepsis (32). Accelerated apoptosis-induced loss of immune cells is an important trigger of the immunosuppressive phase during sepsis (1).

It is well established that immune cells undergo massive and apparently unregulated apoptosis in septic patients and animals $(1,11)$. Sepsis impairs immune function by inducing widespread apoptosis in the spleen, thymus, lymph nodes, and other organs (16). Deregulated apoptosis causes immunosuppression during sepsis, due to extensive lymphocyte loss and to a potential immunosuppressive effect of surviving immune effector cells, such as DCs (19).

In experimental sepsis models, with the exception of the thymus, the majority of lymphoid tissues do not show marked evidence of apoptosis until a late time point ( $>12 \mathrm{~h}$ ) following initiation of infection (33). Autopsy studies in septic patients reported a marked, progressive apoptotic loss of immune cells $(1,17)$. Lymphocytes and DCs were markedly depleted in lymphoid organs with sepsis, and $>3 \%$ of cells exhibited histological signs of apoptosis. The loss in splenic lymphocytes was associated with a significant reduction in circulating lymphocytes, which led to subsequent failure of the adaptive and innate immune systems $(18,34)$. In septic patients, apoptotic lymphocytes were positive for active caspases 8 and 9 , consistent with death occurring by intrinsic and extrinsic apoptotic signaling pathways (31).

The potential importance of apoptosis has been indicated by the following studies demonstrating that prevention of immune cell apoptosis improves survival in experimental animal models of sepsis. Conversely, the adoptive transfer of apoptotic cells caused immunosuppression and worsened survival in a murine septic model, which was interferon (IFN)-dependent (35).

Lymphocytes and myeloid antigen-presenting cell (APC) apoptosis. Current data indicates that an increased level of apoptosis in lymphocytes and DCs contributes to immunosuppression during sepsis, which places the patient at risk of secondary infections $(18,36)$. Direct apoptotic depletion of B lymphocytes, CD4 ${ }^{+} \mathrm{T}$ lymphocytes and DCs, may lead to subsequent failure of the adaptive and innate immune systems, which places the patient at risk of nosocomial infec- 
tions $(17,18)$. Interventions aimed at reducing lymphocyte and DC apoptosis may improve survival in animal endotoxemia models $(12,36)$.

Lymphocytes are central to the adaptive immune response and rapidly expand in response to cytokines and antigen-specific stimulation (37). Apoptosis of lymphocytes has been observed in animal models and in autopsies of septic patients (1,33). Lymphocyte apoptosis is associated with immune dysfunction as a result of the decreased proliferation and IFN release capability $(38,39)$. Lymphocyte-deficient mice treated with caspase inhibitors did not have improved survival in sepsis, which demonstrated that the presence of lymphocytes is a prerequisite for their efficacy $(38,39)$. The marked reduction in the numbers of $\mathrm{T}$ and $\mathrm{B}$ cells lead to an impaired inflammatory response against opportunistic pathogens.

DCs are a group of APCs that have the capacity to interact with $\mathrm{T}$ and $\mathrm{B}$ cells and modulate their responses to invading pathogens (40). The depletion of DCs is potentially associated with failure to induce a protective Th1 immune response, which is predictive of fatal outcome in septic patients (41). Sepsis causes a marked reduction in the percentage area of the spleen occupied by follicular DCs (42). A marked apoptosis-induced loss of follicular DCs and interdigitating DCs significantly compromises B and T cell function, impairs the ability for defense against pathogens and contributes to immune suppression (42).

Loss of $\mathrm{CD}^{+} \mathrm{T}$ lymphocytes limits macrophage activation and impairs the correct inflammatory response to the invading organism (17). Loss of DCs in sepsis may significantly impair $\mathrm{B}$ and $\mathrm{T}$ cell function (18). Therefore, inhibition of apoptosis has served as a therapeutic target for sepsis.

Inhibition of apoptosis as a therapeutic target for sepsis. Several experimental approaches for suppressing apoptosis protect mice from sepsis lethality, further supporting a causative role of apoptosis for the pathogenesis and/or progression of sepsis (12).

Pharmacological interventions have also been used to prevent the initiation of apoptosis in murine models of sepsis. For example, pan caspase inhibitor (38) or specific caspase-3 inhibitor (39) decreased apoptosis and improved survival in a murine cecal ligation and puncture (CLP) model. Furthermore, small interfering (si)RNA knockdown of caspase 8 (43), Bim and p53 upregulated modulator of apoptosis (44), as well as genetic deficiency of caspase 7 (45), prevented sepsis-induced lymphocyte apoptosis, decreased systemic bacterial counts, and improved prognosis. These protective effects were associated with matured lymphocytes, as lymphocyte-deficient mice did not experience any benefit from caspase inhibition $(38,39)$.

Transgenic mice overexpressing the anti-apoptotic, pro-survival protein B cell lymphoma 2 (Bcl-2) in T lymphocytes were protected against $\mathrm{T}$ lymphocyte apoptosis and partly protected against B lymphocyte apoptosis, which significantly improve survival $(46,47)$. In addition, adoptive transfer of Bcl-2 overexpressing $\mathrm{T}$ cells may also improve the outcome $(46,47)$.

Therapeutic strategies aimed at reducing DC and macrophages apoptosis may also improve survival in animal endotoxemia models. The prevention of cellular synthesis of Fas or FasL and the administration of an inhibitor of Fas-FasL binding have shown benefit in a murine CLP study with decreased apoptotic cell death of peritoneal, splenic macrophages and Kupffer cells (48).

The inhibition of Fas-FasL signaling, caspase inhibition (pharmacological or genetic), and the overexpression of downstream anti-apoptotic molecules (Bcl-2) improve the survival of septic mice (46). These studies employing various methods of immune cell apoptosis inhibition provide compelling evidence that immunosuppression resulting from the loss of lymphocytes and DCs is a central pathogenic event in sepsis $(46,47)$. These results suggest possible novel targets for the treatment of sepsis.

Potential limitations of anti-apoptotic therapy. As discussed above, therapeutic strategies aimed at reducing apoptosis could improve the outcome in animal models. Unfortunately, these treatments have yet to be used in clinical trials partly due to the potential limitations of anti-apoptotic therapy.

Firstly, successful anti-apoptotic therapy may be clinically challenging. Small quantities of uninhibited caspase-3 were sufficient to initiate apoptosis (30). Therefore, it would be difficult to get nearly complete inhibition of active caspase-3 (49). In addition, the protective effect of caspase inhibitor in sepsis was lost at high doses (38).

Secondly, deliberate complete inhibition of apoptosis may have unexpected and undesired consequences. Although caspase activation is assumed to be synonymous with cell death, it is now clear that caspases are multifunctional enzymes that have numerous functions, including regulation of proliferation and differentiation (50). For instance, caspase inhibition may affect lymphocyte activation, proliferation and protective immunity (51). In addition, certain intracellular agents may use host cell apoptosis for their own replication (52).

Therefore, the beneficial effects of caspase inhibition could be counter-balanced by its adverse effects. Instead of directly inhibiting immune cell apoptosis, the restoration of immune homeostasis may serve as a novel and effective therapeutic approach.

\section{Immune cell autophagy in sepsis}

Regulatory mechanisms and signaling pathways of autophagy. Although apoptosis has been well-studied, another cellular signaling pathway, autophagy, and its association with inflammation and immunity has attracted increasing interest (53). Autophagy is a catabolic process whereby cells respond to energy stress by recycling intracellular components: Proteins, ribosomes, lipids, and entire organelles $(53,54)$.

The autophagic process involves the vesicular sequestration of cytoplasmic proteins, organelles or pathogens, resulting in a double-membrane vesicle formation, termed an autophagosome. This is followed by the fusion of the autophagosome with a lysosome to form an autolysosome, in which the captured material, together with the inner membrane, is degraded (54).

A low level of constitutive autophagy occurs under normal physiological conditions. At a basal rate, autophagy functions as a housekeeping mechanism underlying the maintenance of normal cellular homoeostasis (54). Upon extracellular or intracellular stress and signals, autophagy may be markedly upregulated to function as a self-destructive 
signaling pathway that promotes cell survival in an adverse environment. As a major cellular catabolic system targeting a variety of substrates, the autophagic process requires regulation $(54,55)$. Various types of autophagy-associated (Atg) proteins are involved in these steps and comprise the core autophagic machinery (53).

An efficient mechanism underlying the induction of autophagy is crucial for organisms to adapt to stress and extracellular signals. A central inhibitor of autophagy is the serine/threonine protein kinase target of rapamycin TOR. Mammalian (m)TOR interacts with, phosphorylates, and inactivates Unc 51 like autophagy activating kinases (ULKs) and Atg13 (56). Upon mTOR inhibition by starvation or rapamycin, ULK1/2 are activated and phosphorylate Atg13 and FAK family kinase interacting protein of $200 \mathrm{kDa}$, which are crucial for autophagic activity (56).

The formation of sequestering vesicles is the most complex step of autophagy. The nucleation and assembly of the initial phagophore membrane requires the class III phosphatidylinositol 3-kinase complex, which is composed of Vps34, Vps15, Atg14 and Beclin 1. The function of Beclin 1 in autophagy is regulated by $\mathrm{Bcl}-2$, which inhibits autophagy by binding and sequestering Beclin 1; the dissociation of Beclin 1 from Bcl-2 is required for autophagy induction (57).

Two ubiquitin-like conjugation systems are required for autophagosome elongation and closure. In one ubiquitin-like signaling cascade, Atg7 and 10 conjugates Atg12 to Atg5. The Atg5-Atg12 conjugate then binds Atg16 1. In the other ubiquitin-like signaling cascade, light chain 3 I (LC3-I) is conjugated to lipid phosphatidyl ethanolamine by Atg 3 and Atg7. Functionally, LC3-II is important for the elongation and closure of autophagosomes (58). In addition, LC3-II binds to the adaptor protein p62/sequestosome 1 that is involved in trafficking proteins to the proteasome and serves to facilitate the autophagic degradation of ubiquitinated protein aggregates (58). In mammalian cells, this fusion event requires the lysosomal membrane protein lysosomal associated membrane protein 2 (59).

Function of autophagy in infectious diseases and immune responses. Recently, immunologists have identified a number of immunological processes that are dependent upon cellular autophagy, including antigen presentation, lymphocyte development and function, pathogen recognition and elimination, and inflammatory regulation (53-55).

Elimination of intracellular microbes. The ability of autophagy to remove large cytoplasmic structures with selectivity enables this signaling pathway to be used to clear intracellular microbes. Recently, an increasing number of studies have been conducted on the antimicrobial effects of autophagy and its potential relevance to human immunity (60). Autophagy degrades intracellular pathogens, including bacteria, virus, fungi and protozoa in a selective form of autophagy, termed xenophagy. Numerous medical pathogens may be degraded by xenophagy, including bacteria such as group A Streptococcus, Mycobacterium, Shigella flexneri, Salmonella enterica, Listeria monocytogenes, Francisella tularensis, viruses such as herpes simplex virus-1, and parasites such as Toxoplasma gondii (61).
Maintenance of lymphocyte homeostasis. Recent studies have investigated the critical role of autophagy in lymphocyte development and function $(59,62,63)$. Specifically, the coordinated regulation of autophagy and apoptosis is essential for the maintenance of lymphocyte homeostasis. Autophagy supports proliferation and survival during the transition between naive and effector lymphocytes (64). As lymphocytes mature, autophagy regulates the reduction in mitochondria that occurs in naive lymphocytes (59).

A recent study investigated the important role of Atg5 in lymphocyte development and function. Atg5 deficient CD4 ${ }^{+}$ and $\mathrm{CD}^{+} \mathrm{T}$ cells failed to repopulate the periphery due to high levels of cell death and failure to undergo efficient proliferation following $\mathrm{T}$ cell receptor stimulation (64). Atg7 and Atg5 are required in a cell-intrinsic manner for the survival of mature primary $\mathrm{T}$ lymphocytes. This demonstrated that autophagy eliminates superfluous mitochondria in $\mathrm{T}$ lymphocytes as part of normal $\mathrm{T}$ cell homeostasis (62).

Similar to T lymphocyte development, B lymphocytes require autophagy during development and for maintenance in the periphery. Atg5-deficient pro-B cells do not efficiently develop into pre-B cells, but instead die following increased apoptosis (63).

\section{Regulation of immunity and inflammation}

There is a complex reciprocal association between autophagy signaling pathway/proteins and immunity and inflammation $(54,55)$. Immune and inflammatory signals do not function solely in the induction and suppression of autophagy, in addition, the autophagic machinery also participates in the regulation of immunity and inflammation (53).

Immune signaling molecules, including pathogen associated molecular patterns such as lipopolysaccharide (LPS) and lymphotoxin $\alpha$, and damage-associated molecular patterns (DAMPs) such as ATP and reactive oxygen species (ROS) may induce autophagy $(21,53)$.

The autophagic machinery is necessary for DCs to process and present extracellular microbial antigens for major histocompatibility complex (MHC) class II presentation in vivo. Intracellular antigens are engulfed by autophagosomes, transported to MHC class II loading compartments, and loaded onto MHC class II molecules for presentation to $\mathrm{CD}^{+} \mathrm{T}$ cells (65). Therefore, in the absence of Atg5 in DCs, the animal failed to induce adequate Th1 cell immunity (65).

Autophagy regulates inflammatory signaling during inflammasome activation (53). Autophagy may avoid unscheduled inflammasome activation by ROS or mitochondrial (mt)DNA and deliver inflammasome substrates via an autophagy-based secretory signaling pathway (21). It has been demonstrated that LPS-induced cytokine production is regulated by autophagy. The inhibition of autophagy significantly increased the production of TNF- $\alpha$ and IL-1 $\beta$ (65), whereas induction of autophagy reduced serum levels of IL-1 $\beta$ and IL-18 in response to LPS stimulation (53).

Deficiencies in autophagy promoted the accumulation of dysfunctional mitochondrial and cytosolic translocation of mtDNA in response to LPS in macrophages. Cytosolic mtDNA contributed to the secretion of IL-1 $\beta$ and IL-18 in response to LPS and ATP (21). Circulating mtDNA occurs in patients with 
sepsis and correlates with disease severity (66), and is able to activate immunity (67).

Dupont et al (68) reported that autophagy contributed to the biogenesis and secretion of the pro-inflammatory cytokine IL-1 $\beta$. This suggested that the autophagy-based secretory signaling pathway participated in the extracellular delivery of numerous cytosolic proteins via the Golgi apparatus $(68,69)$. Autophagy-based secretion of cytokines may significantly influence the activation and duration of inflammation.

The efficient clearance of apoptotic debris prevents secondary necrosis, which releases DAMPs that trigger inflammation (70). Autophagy also negatively regulates the induction of type I IFN secretion via the autophagic elimination of dysfunctional mitochondria and reduction of ROS (71).

\section{Reciprocal regulation between apoptosis and autophagy}

Apoptotic cell death is considered to be a mechanism underlying immunosuppression and multiple organ dysfunctions following sepsis $(8,11)$. Another cellular process, autophagy, is also activated in sepsis and serves a protective role by maintaining cellular homeostasis and limiting cell damage and death. The association between autophagy ('self-eating') and apoptosis ('self-killing') is complex, and remains the subject of extensive ongoing studies $(53,54)$. These two cellular processes share common components, regulate and modify the activity of one another, and can be triggered by common stimuli including starvation, oxidative stress, and cytokines $(72,73)$.

The three processes by which autophagy regulates apoptosis are as follows: i) Regulation of apoptosis by specific autophagy proteins; ii) activation of caspases on autophagosome membranes, which is dependent on autophagosome formation; iii) and regulation of apoptosis by autophagic degradation requires autophagosome formation and lysosome activity (74). The two mechanistic processes by which apoptosis regulates autophagy are the following: i) Direct regulation of autophagy by specific apoptotic proteins; and ii) regulation of autophagy by activated caspases, which necessitates the activation of the apoptotic process as a whole (74).

The anti-apoptotic protein Bcl-2 regulates autophagy and apoptosis by binding the pro-autophagic proteins Beclin1 and Bcl 2 associated $\mathrm{X}$ protein (and others). The Bcl-2-binding domain of Beclin1 serves as a point of crosstalk between autophagic and apoptotic signaling pathways at the level of autophagy induction (75). Beclin $1 / \mathrm{Bcl}-2$ interaction has recently been shown to negatively regulate autophagy, providing evidence for the association between autophagy and apoptosis (76).

It has been demonstrated that mitochondria may undergo autophagy, which may be detrimental for the switch between autophagy and apoptosis (28). The term mitophagy is used to refer to mitochondrial degradation by autophagy. This type of selective process is thought to serve a crucial function in mitochondrial homeostasis (77). Mitophagy inhibition leads to the accumulation of damaged, ROS-generating mitochondria, which promotes intrinsic apoptotic cell death (78). In addition, the localization of numerous autophagic mitochondrial regulators, the integration of mitochondria in several signaling networks and their potential to modulate these signaling pathways suggest a significant mitochondrial influence on autophagy (77).
Tang et al (79) demonstrated that reduced exogenous high mobility group box 1 (HMGB1) increases autophagy and oxidized HMGB1 increases apoptosis. HMGB1 is required for binding to Beclin 1 and sustaining autophagy. It has been suggested that HMGB1 effects the crosstalk between apoptosis and autophagy (79).

Atg5 and Atg12 are integral parts of the autophagic core machinery and are crucial for the induction of autophagy (58). In addition, Atg5 and Atg12 were demonstrated to serve important functions in the initiation of apoptosis (28). Overexpression of Atg5 may induce autophagy and enhance the susceptibility of the intrinsic cell death signaling pathway in tumor cells (80). However, Atg5 can interact directly with Fas (TNFRSF6) associated via death domain, thereby stimulating caspase-dependent death (81). Therefore, Atg5 may trigger apoptosis via a number of mechanisms and form part of the molecular mechanisms underlying the inhibitory crosstalk between apoptosis and autophagy.

Autophagy frequently precedes apoptosis, and usually serves as a last survival attempt prior to cell death (28). The inhibition of autophagy leads to an increased susceptibility to apoptotic stimulation, whereas increased autophagy improves cell survival by inhibiting apoptosis $(81,82)$. The balance between apoptosis and autophagy may influence cellular homeostasis in the progression of sepsis $(9,10)$. Since the potential benefits of apoptosis inhibition may be compromised by its unforeseen side effects, it was hypothesized that the apoptotic-autophagic balance may be restored by inducing autophagy $(9,10)$. Autophagy, which exerts inhibition on immune cell apoptosis, may re-engage host immune function and may avoid the side effects of complete inhibition of apoptosis $(9,10)$. The interaction between autophagy and apoptosis, as well as the effects on immune cell responses during sepsis, may uncover novel therapeutic approaches for the detection and treatment of sepsis.

\section{Autophagy as a therapeutic target in pulmonary diseases}

Previous studies have attempted to manipulate autophagy therapeutically $(53-55,60)$. Autophagy has become a therapeutic target in a variety of pulmonary diseases, including infectious disease, chronic obstructive pulmonary disease (COPD), pulmonary vascular hypertension, idiopathic pulmonary fibrosis and lung cancer $(83,84)$.

Pharmacological or immunological activation of autophagy can enhance microbial elimination. Several therapies that stimulate autophagy have previously been reported to be effective against Mycobacterium tuberculosis infection (85). Autophagy has also been described as a mechanism underlying the defense against other respiratory pathogens. Genetic deficiency of autophagic genes promoted Legionella pneumophila (86), Klebsiella pneumoniae (87), growth and influenza virus replication (88).

In a mouse model, chronic exposure to hypoxia resulted in an increased incidence of pulmonary hypertension $(\mathrm{PH})$, which was associated with increased autophagosome formation in lung tissue. Autophagy deficient, light chain 3B (LC3B) null mice displayed heightened indices of $\mathrm{PH}$ following exposure to chronic hypoxia compared with wild-type mice (89). 
Lung tissue samples from patients with idiopathic pulmonary fibrosis and human lung fibroblasts treated with TGF- $\beta$ demonstrated increased cellular senescence and decreased autophagic activity. Treatment with the autophagy inducer rapamycin partially protected against lung fibrosis (90).

In COPD lung tissue samples, an elevation of general autophagy markers and the increased occurrence of autophagosomes in situ was observed. The inhibition of autophagy by LC3B-knockdown protected epithelial cells from cigarette smoke extract (CSE)-induced apoptosis and airspace enlargement (91). The induction of autophagy at early stages of COPD progression suggests novel therapeutic targets for the treatment of CSE-induced lung injury $(91,92)$.

Autophagy inhibition sensitizes tumor cells against cell death induced by diverse cytotoxic agents. Accordingly, there are numerous phase I/II clinical trials in progress using chloroquine or hydroxychloroquine in combination with chemotherapy for the treatment of a range of hematological and solid tumors (93). Chloroquine inhibits autophagy-mediated cell survival in tumor cells, and was used as an intervention for patients with small cell lung cancer in a clinical trial (phase I). In addition, therapies using hydrochloroquine and other anticancer drugs have been used for non-small cell lung cancer (NSCLC) in two clinical trials (phase I/II and II) (93). Conversely, the induction of autophagy via mTOR inhibitors has been associated with radio-sensitization in NSCLC cells (94).

\section{Autophagy as a potential therapeutic target for sepsis}

Autophagy is emerging as an attractive therapeutic target for numerous diseases, including infectious diseases. Autophagy can be stimulated by multiple cellular stresses, such as ROS, protein aggregates, damaged organelles, and intracellular pathogens $(53,54)$. All of these cellular stresses occur during the progression of sepsis $(53,54)$. Recent studies have demonstrated that autophagy is induced in patients with sepsis and in the clinically relevant CLP animal model of sepsis $(23,83)$.

As an adaptive response, autophagy has been demonstrated to protect against sepsis-induced multiple organ dysfunctions, including acute liver injury $(22,23)$, acute kidney injury $(24,25)$, myocardial dysfunction $(95)$, acute lung injury $(25,26)$ and immune dysfunction $(9,10)$. An impairment of autophagic function may worsen multiple organ dysfunctions.

Transgenic mice overexpressing the LC3 gene exhibited increased clearance of autophagosomes and improved survival following CLP. This protective effect was associated with decreased rates of apoptotic cell death, inflammatory responses, neutrophil accumulation, albumin leakage, and edema formation (26). Conversely, autophagy-deficient mice produce increased caspase-1-dependent cytokines and show greater susceptibility to LPS administration, indicating a protective role for autophagy in sepsis (21).

Sepsis or LPS-induced autophagy protects against hepatocellular death both in vivo and in vitro. Inhibition of autophagy using 3-methyladenine or siRNA specific to VPS34 further increases sepsis or LPS-induced hepatocyte apoptosis (22). Autophagy deficiency increases TNF-induced apoptotic cell death, depletion of ATP and reduction of albumin production in hepatocytes. In addition, the inhibition of autophagic flux by chloroquine administration immediately following CLP resulted in elevated serum transaminase levels and a significant increase in mortality (23).

Specific knockdown of Atg7 increases sepsis-induced acute kidney dysfunction. These results demonstrated that a decline in autophagy may contribute to proximal tubular dysfunction, whereas the maintenance of sufficient autophagy prevents apoptotic cell death (24). Pharmacological induction of autophagy, even following established endotoxemia, has therapeutic utility in facilitating renal recovery (25).

Incomplete autophagic flux may contribute to sepsis-induced cardiac dysfunction. Rapamycin induced complete activation of autophagy, restored CLP-induced depressed cardiac performances, and served a cardioprotective role in sepsis. This cardioprotective effect was also associated with increased ATP levels, and decreased inflammatory responses (95).

The downregulation of autophagy may lead to systemic inflammation and acute lung injury in sepsis. The direct modification of autophagy using rapamycin resulted in improved lung injury (27). LC3 transgene attenuates lung injury and inflammation in sepsis, possibly by increasing the clearance of autophagosomes (26).

The depletion of autophagic proteins promoted the accumulation of dysfunctional mitochondria and cytosolic translocation of mtDNA in response to LPS and ATP in macrophages (21). Our previous study demonstrated that autophagy inhibition by 3-MA or Beclin-1 siRNA treatment inhibited LPS-induced autophagic flux while inducing apoptotic signaling in macrophages (9). These results provide further evidence that the autophagic response is a protective mechanism in LPS-stimulated macrophages and splenocytes $(9,10)$.

Combined with recent studies investigating autophagy in sepsis, the present review highlighted the therapeutic potential of autophagy activation for multiple organ dysfunctions in septic patients. All the reported experiments provide potential therapies that could activate autophagy during sepsis.

\section{Conclusion}

Sepsis remains a major but largely under-recognized healthcare problem worldwide. Accelerated apoptosis of immune cells leads to immunosuppression. Sepsis-induced immunosuppression occurs at a later phase, thereby making it an attractive therapeutic target. Another cellular process, autophagy, protects against sepsis-induced MOF and immune dysfunction.

Notably, the reciprocal regulation between autophagy and apoptosis may influence cellular fate. The induction of autophagy may inhibit immune cell apoptosis and re-engage host immune homeostasis, and avoid the side effects associated with the complete inhibition of apoptosis. From a therapeutic perspective, rectification of the apoptotic-autophagic balance in immune cells may serve as a novel therapeutic approach for the treatment of septic patients. Whether autophagy represents a useful target in sepsis-induced immunosuppression will need to be addressed by further experiments and clinical trials. 


\section{Acknowledgements}

The present study was supported by a grant from the National Natural Science Foundation of China (grant no. 81401631).

\section{References}

1. Hotchkiss RS, Swanson PE, Freeman BD, Tinsley KW, Cobb JP, Matuschak GM, Buchman TG and Karl IE: Apoptotic cell death in patients with sepsis, shock, and multiple organ dysfunction. Crit Care Med 27: 1230-1251, 1999.

2. Aneja R and Fink MP: Promising therapeutic agents for sepsis. Trends Microbiol 15: 31-37, 2007.

3. Angus DC, Linde-Zwirble WT, Lidicker J, Clermont G, Carcillo J and Pinsky MR: Epidemiology of severe sepsis in the United States: Analysis of incidence, outcome, and associated costs of care. Crit Care Med 29: 1303-1310, 2001.

4. Angus DC and van der Poll T: Severe sepsis and septic shock. N Engl J Med 369: 840-851, 2013.

5. Ziegler EJ, Fisher CJ Jr., Sprung CL, Straube RC, Sadoff JC, Foulke GE, Wortel CH, Fink MP, Dellinger RP, Teng NN, et al: Treatment of gram-negative bacteremia and septic shock with HA-1A human monoclonal antibody against endotoxin. A randomized, double-blind, placebo-controlled trial. The HA-1A Sepsis Study Group. N Engl J Med 324: 429-436, 1991.

6. Fisher CJ Jr, Slotman GJ, Opal SM, Pribble JP, Bone RC, Emmanuel G, Ng D, Bloedow DC and Catalano MA; IL-1RA Sepsis Syndrome Study Group: Initial evaluation of human recombinant interleukin-1 receptor antagonist in the treatment of sepsis syndrome: A randomized, open-label, placebo-controlled multicenter trial. Crit Care Med 22: 12-21, 1994.

7. Fisher CJ Jr, Agosti JM, Opal SM, Lowry SF, Balk RA, Sadoff JC, Abraham E, Schein RM and Benjamin E: Treatment of septic shock with the tumor necrosis factor receptor: Fc fusion protein. The Soluble TNF Receptor Sepsis Study Group. N Engl J Med 334: 1697-1702, 1996.

8. Boomer JS, Green JM and Hotchkiss RS: The changing immune system in sepsis: Is individualized immuno-modulatory therapy the answer? Virulence 5: 45-56, 2014.

9. Zhang L, Cardinal JS, Bahar R, Evankovich J, Huang H, Nace G, Billiar TR, Rosengart MR, Pan P and Tsung A: Interferon regulatory factor-1 regulates the autophagic response in LPS-stimulated macrophages through nitric oxide. Mol Med 18: 201-208, 2012.

10. Zhang L, Cardinal JS, Pan P, Rosborough BR, Chang Y, Yan W, Huang H, Billiar TR, Rosengart MR and Tsung A: Splenocyte apoptosis and autophagy is mediated by interferon regulatory factor 1 during murine endotoxemia. Shock 37: 511-517, 2012.

11. Hotchkiss RS and Karl IE: The pathophysiology and treatment of sepsis. N Engl J Med 348: 138-150, 2003.

12. Hotchkiss RS and Nicholson DW: Apoptosis and caspases regulate death and inflammation in sepsis. Nat Rev Immunol 6: 813-822, 2006.

13. Venet F, Chung CS, Monneret G, Huang X, Horner B, Garber M and Ayala A: Regulatory T cell populations in sepsis and trauma. J Leukoc Biol 83: 523-535, 2008.

14. Delano MJ, Scumpia PO, Weinstein JS, Coco D, Nagaraj S, Kelly-Scumpia KM, O'Malley KA, Wynn JL, Antonenko S, Al-Quran SZ, et al: MyD88-dependent expansion of an immature GR-1(+)CD11b $(+)$ population induces T cell suppression and Th2 polarization in sepsis. J Exp Med 204: 1463-1474, 2007.

15. Osuchowski MF, Welch K, Siddiqui J and Remick DG: Circulating cytokine/inhibitor profiles reshape the understanding of the SIRS/CARS continuum in sepsis and predict mortality. J Immunol 177: 1967-1974, 2006.

16. Adib-Conquy $\mathrm{M}$ and Cavaillon JM: Compensatory anti-inflammatory response syndrome. Thromb Haemost 101: 36-47, 2009.

17. Hotchkiss RS, Tinsley KW, Swanson PE, Schmieg RE Jr, Hui JJ, Chang KC, Osborne DF, Freeman BD, Cobb JP, Buchman TG and Karl IE: Sepsis-induced apoptosis causes progressive profound depletion of B and CD4+ T lymphocytes in humans. J Immunol 166: 6952-6963, 2001.

18. Hotchkiss RS, Tinsley KW, Swanson PE, Grayson MH, Osborne DF, Wagner TH, Cobb JP, Coopersmith C and Karl IE: Depletion of dendritic cells, but not macrophages, in patients with sepsis. J Immunol 168: 2493-2500, 2002.
19. Stearns-Kurosawa DJ, Osuchowski MF, Valentine C, Kurosawa S and Remick DG: The pathogenesis of sepsis. Annu Rev Pathol 6: 19-48, 2011

20. Levine B and Yuan J: Autophagy in cell death: An innocent convict? J Clin Invest 115: 2679-2688, 2005.

21. Nakahira K, Haspel JA, Rathinam VA, Lee SJ, Dolinay T, Lam HC, Englert JA, Rabinovitch M, Cernadas M, Kim HP, et al: Autophagy proteins regulate innate immune responses by inhibiting the release of mitochondrial DNA mediated by the NALP3 inflammasome. Nat Immunol 12: 222-230, 2011.

22. Carchman EH, Rao J, Loughran PA, Rosengart MR and Zuckerbraun BS: Heme oxygenase-1-mediated autophagy protects against hepatocyte cell death and hepatic injury from infection/sepsis in mice. Hepatology 53: 2053-2062, 2011.

23. Takahashi W, Watanabe E, Fujimura L, Watanabe-Takano H, Yoshidome H, Swanson PE, Tokuhisa T, Oda S and Hatano M: Kinetics and protective role of autophagy in a mouse cecal ligation and puncture-induced sepsis. Crit Care 17: R160, 2013.

24. Hsiao HW, Tsai KL, Wang LF, Chen YH, Chiang PC, Chuang SM and Hsu C: The decline of autophagy contributes to proximal tubular dysfunction during sepsis. Shock 37: 289-296, 2012.

25. Howell GM, Gomez H, Collage RD, Loughran P, Zhang X, Escobar DA, Billiar TR, Zuckerbraun BS and Rosengart MR: Augmenting autophagy to treat acute kidney injury during endotoxemia in mice. PLoS One 8: e69520, 2013.

26. Lo S, Yuan SS, Hsu C, Cheng YJ, Chang YF, Hsueh HW, Lee PH and Hsieh YC: Lc3 over-expression improves survival and attenuates lung injury through increasing autophagosomal clearance in septic mice. Ann Surg 257: 352-363, 2013.

27. Yen YT, Yang HR, Lo HC, Hsieh YC, Tsai SC, Hong CW and Hsieh $\mathrm{CH}$ : Enhancing autophagy with activated protein $\mathrm{C}$ and rapamycin protects against sepsis-induced acute lung injury. Surgery 153: 689-698, 2013.

28. Hsieh YC, Athar M and Chaudry IH: When apoptosis meets autophagy: Deciding cell fate after trauma and sepsis. Trends Mol Med 15: 129-138, 2009.

29. Hengartner MO: The biochemistry of apoptosis. Nature 407: $770-776,2000$

30. Budihardjo I, Oliver H,Lutter M, Luo X and Wang X: Biochemical pathways of caspase activation during apoptosis. Annu Rev Cell Dev Biol 15: 269-290, 1999.

31. Hotchkiss RS, Osmon SB, Chang KC, Wagner TH, Coopersmith CM and Karl IE: Accelerated lymphocyte death in sepsis occurs by both the death receptor and mitochondrial pathways. J Immunol 174: 5110-5118, 2005.

32. Boomer JS, To K, Chang KC, Takasu O, Osborne DF, Walton AH, Bricker TL, Jarman SD II, Kreisel D, Krupnick AS, et al: Immunosuppression in patients who die of sepsis and multiple organ failure. JAMA 306: 2594-2605, 2011.

33. Wesche DE, Lomas-Neira JL, Perl M, Chung CS and Ayala A: Leukocyte apoptosis and its significance in sepsis and shock. J Leukoc Biol 78: 325-337, 2005 .

34. Felmet KA, Hall MW, Clark RS, Jaffe R and Carcillo JA: Prolonged lymphopenia, lymphoid depletion, and hypoprolactinemia in children with nosocomial sepsis and multiple organ failure. J Immunol 174: 3765-3772, 2005.

35. Hotchkiss RS, Chang KC, Grayson MH, Tinsley KW, Dunne BS, Davis CG, Osborne DF and Karl IE: Adoptive transfer of apoptotic splenocytes worsens survival, whereas adoptive transfer of necrotic splenocytes improves survival in sepsis. Proc Natl Acad Sci USA 100: 6724-6729, 2003.

36. Wesche-Soldato DE, Swan RZ, Chung CS and Ayala A: The apoptotic pathway as a therapeutic target in sepsis. Curr Drug Targets 8: 493-500, 2007.

37. Springer TA: Traffic signals on endothelium for lymphocyte recirculation and leukocyte emigration. Annu Rev Physiol 57: 827-872, 1995

38. Hotchkiss RS, Tinsley KW, Swanson PE, Chang KC, Cobb JP, Buchman TG, Korsmeyer SJ and Karl IE: Prevention of lymphocyte cell death in sepsis improves survival in mice. Proc Natl Acad Sci USA 96: 14541-14546, 1999.

39. Hotchkiss RS, Chang KC, Swanson PE, Tinsley KW, Hui JJ, Klender P, Xanthoudakis S, Roy S, Black C, Grimm E, et al: Caspase inhibitors improve survival in sepsis: A critical role of the lymphocyte. Nat Immunol 1: 496-501, 2000.

40. Dong X, Shen K and Bulow HE: Intrinsic and extrinsic mechanisms of dendritic morphogenesis. Annu Rev Physiol 77: 271-300, 2015 
41. Gautier EL, Huby T, Saint-Charles F, Ouzilleau B, Chapman MJ and Lesnik P: Enhanced dendritic cell survival attenuates lipopolysaccharide-induced immunosuppression and increases resistance to lethal endotoxic shock. J Immunol 180: 6941-6946, 2008.

42. Tinsley KW, Grayson MH, Swanson PE, Drewry AM, Chang KC, Karl IE and Hotchkiss RS: Sepsis induces apoptosis and profound depletion of splenic interdigitating and follicular dendritic cells. J Immunol 171: 909-914, 2003.

43. Wesche-Soldato DE, Chung CS, Lomas-Neira J, Doughty LA, Gregory SH and Ayala A: In vivo delivery of caspase- 8 or Fas siRNA improves the survival of septic mice. Blood 106: 2295-2301, 2005.

44. Brahmamdam P, Watanabe E, Unsinger J, Chang KC, Schierding W, Hoekzema AS, Zhou TT, McDonough JS Holemon $\mathrm{H}$, Heidel JD, et al: Targeted delivery of siRNA to cell death proteins in sepsis. Shock 32: 131-139, 2009.

45. Lamkanfi M, Moreira LO, Makena P, Spierings DC, Boyd K, Murray PJ, Green DR and Kanneganti TD: Caspase-7 deficiency protects from endotoxin-induced lymphocyte apoptosis and improves survival. Blood 113: 2742-2745, 2009.

46. Hotchkiss RS, Swanson PE, Knudson CM, Chang KC, Cobb JP, Osborne DF, Zollner KM, Buchman TG, Korsmeyer SJ and Karl IE: Overexpression of Bcl-2 in transgenic mice decreases apoptosis and improves survival in sepsis. J Immunol 162 4148-4156, 1999.

47. Peck-Palmer OM, Unsinger J, Chang KC, McDonough JS, Perlman H, McDunn JE and Hotchkiss RS: Modulation of the Bcl-2 family blocks sepsis-induced depletion of dendritic cells and macrophages. Shock 31: 359-366, 2009.

48. Chung CS, Song GY, Lomas J, Simms HH, Chaudry IH and Ayala A: Inhibition of Fas/Fas ligand signaling improves septic survival: Differential effects on macrophage apoptotic and functional capacity. J Leukoc Biol 74: 344-351, 2003.

49. Méthot N, Huang J, Coulombe N, Vaillancourt JP, Rasper D, Tam J, Han Y, Colucci J, Zamboni R, Xanthoudakis S, et al: Differential efficacy of caspase inhibitors on apoptosis markers during sepsis in rats and implication for fractional inhibition requirements for therapeutics. J Exp Med 199: 199-207, 2004.

50. Kidd VJ: Proteolytic activities that mediate apoptosis. Annu Rev Physiol 60: 533-573, 1998.

51. Martinon F and Tschopp J: Inflammatory caspases: Linking an intracellular innate immune system to autoinflammatory diseases. Cell 117: 561-574, 2004.

52. Parrino J, Hotchkiss RS and Bray M: Prevention of immune cell apoptosis as potential therapeutic strategy for severe infections. Emerg Infect Dis 13: 191-198, 2007.

53. Levine B, Mizushima N and Virgin HW: Autophagy in immunity and inflammation. Nature 469: 323-335, 2011.

54. Klionsky DJ and Codogno P: The mechanism and physiological function of macroautophagy. J Innate Immun 5: 427-433, 2013.

55. He C and Klionsky DJ: Regulation mechanisms and signaling pathways of autophagy. Annu Rev Genet 43: 67-93, 2009.

56. Jung $\mathrm{CH}$, Ro SH, Cao J, Otto NM and Kim DH: mTOR regulation of autophagy. FEBS Lett 584: 1287-1295, 2010.

57. Pattingre S, Tassa A, Qu X, Garuti R, Liang XH, Mizushima N, Packer M, Schneider MD and Levine B: Bcl-2 antiapoptotic proteins inhibit Beclin 1-dependent autophagy. Cell 122: 927-939, 2005

58. Nishida Y, Arakawa S, Fujitani K, Yamaguchi H, Mizuta T, Kanaseki T, Komatsu M, Otsu K, Tsujimoto Y and Shimizu S: Discovery of Atg5/Atg7-independent alternative macroautophagy. Nature 461: 654-658, 2009.

59. Behrends C, Sowa ME, Gygi SP and Harper JW: Network organization of the human autophagy system. Nature 466: 68-76, 2010.

60. Moy RH and Cherry S: Antimicrobial autophagy: A conserved innate immune response in Drosophila. J Innate Immun 5: 444-455, 2013

61. Levine B and Kroemer G: Autophagy in the pathogenesis of disease. Cell 132: 27-42, 2008

62. Pua HH, Guo J, Komatsu M and He YW: Autophagy is essential for mitochondrial clearance in mature T lymphocytes. J Immunol 182: 4046-4055, 2009.

63. Miller BC, Zhao Z, Stephenson LM, Cadwell K, Pua HH Lee HK, Mizushima NN, Iwasaki A, He YW, Swat W and Virgin HW IV: The autophagy gene ATG5 plays an essential role in B lymphocyte development. Autophagy 4: 309-314, 2008.

64. Pua HH, Dzhagalov I, Chuck M, Mizushima N and He YW: A critical role for the autophagy gene Atg5 in T cell survival and proliferation. J Exp Med 204: 25-31, 2007.
65. Lee HK, Mattei LM, Steinberg BE, Alberts P, Lee YH, Chervonsky A, Mizushima N, Grinstein S and Iwasaki A: In vivo requirement for $A \operatorname{tg} 5$ in antigen presentation by dendritic cells. Immunity 32: 227-239, 2010.

66. Pyle A, Burn DJ, Gordon C, Swan C, Chinnery PF and Baudouin SV: Fall in circulating mononuclear cell mitochondrial DNA content in human sepsis. Intensive Care Med 36: 956-962, 2010.

67. Zhang Q, Raoof M, Chen Y, Sumi Y, Sursal T, Junger W, Brohi K, Itagaki K and Hauser CJ: Circulating mitochondrial DAMPs cause inflammatory responses to injury. Nature 464: 104-107, 2010.

68. Dupont N, Jiang S, Pilli M, Ornatowski W, Bhattacharya D and Deretic V: Autophagy-based unconventional secretory pathway for extracellular delivery of IL- 3 . EMBO J 30: 4701-4711, 2011.

69. Jiang S, Dupont N, Castillo EF and Deretic V: Secretory versus degradative autophagy: Unconventional secretion of inflammatory mediators. J Innate Immun 5: 471-479, 2013.

70. Qu X, Zou Z, Sun Q, Luby-Phelps K, Cheng P, Hogan RN, Gilpin $\mathrm{C}$ and Levine B: Autophagy gene-dependent clearance of apoptotic cells during embryonic development. Cell 128: 931-946, 2007.

71. Tal MC, Sasai M, Lee HK, Yordy B, Shadel GS and Iwasaki A: Absence of autophagy results in reactive oxygen species-dependent amplification of RLR signaling. Proc Natl Acad Sci USA 106: 2770-2775, 2009.

72. Maiuri MC, Zalckvar E, Kimchi A and Kroemer G: Self-eating and self-killing: Crosstalk between autophagy and apoptosis. Nat Rev Mol Cell Biol 8: 741-752, 2007.

73. Fimia GM and Piacentini M: Regulation of autophagy in mammals and its interplay with apoptosis. Cell Mol Life Sci 67: 1581-1588, 2010.

74. Rubinstein AD and Kimchi A: Life in the balance-a mechanistic view of the crosstalk between autophagy and apoptosis. J Cell Sci 125: 5259-5268, 2012.

75. Zinkel S, Gross A and Yang E: BCL2 family in DNA damage and cell cycle control. Cell Death Differ 13: 1351-1359, 2006.

76. Shi CS and Kehrl JH: MyD88 and Trif target Beclin 1 to trigger autophagy in macrophages. J Biol Chem 283: 33175-33182, 2008.

77. Kurihara Y, Kanki T, Aoki Y, Hirota Y, Saigusa T, Uchiumi T and Kang D: Mitophagy plays an essential role in reducing mitochondrial production of reactive oxygen species and mutation of mitochondrial DNA by maintaining mitochondrial quantity and quality in yeast. J Biol Chem 287: 3265-3272, 2012.

78. Zhou R, Yazdi AS, Menu P and Tschopp J: A role for mitochondria in NLRP3 inflammasome activation. Nature 469: 221-225, 2011.

79. Tang D, Loze MT, Zeh HJ and Kang R: The redox protein HMGB1 regulates cell death and survival in cancer treatment. Autophagy 6: 1181-1183, 2010.

80. Yousefi S, Perozzo R, Schmid I, Ziemiecki A, Schaffner T, Scapozza L, Brunner T and Simon HU: Calpain-mediated cleavage of Atg 5 switches autophagy to apoptosis. Nat Cell Biol 8: 1124-1132, 2006.

81. Pyo JO, Jang MH, Kwon YK, Lee HJ, Jun JI, Woo HN, Cho DH, Choi B, Lee H, Kim JH, et al: Essential roles of Atg5 and FADD in autophagic cell death: Dissection of autophagic cell death into vacuole formation and cell death. J Biol Chem 280: 20722-20729, 2005.

82. González-Polo RA, Boya P, Pauleau AL, Jalil A, Larochette N, Souquère $S$, Eskelinen EL, Pierron G, Saftig P and Kroemer G: The apoptosis/autophagy paradox: Autophagic vacuolization before apoptotic death. J Cell Sci 118: 3091-3102, 2005.

83. Mizumura K, Cloonan SM, Haspel JA and Choi AM: The emerging importance of autophagy in pulmonary diseases. Chest 142: 1289-1299, 2012.

84. Mizumura K, Choi AM and Ryter SW: Emerging role of selective autophagy in human diseases. Front Pharmacol 5: 244, 2014.

85. Lam KK, Zheng X, Forestieri R, Balgi AD, Nodwell M, Vollett S, Anderson HJ, Andersen RJ, Av-Gay Y and Roberge M: Nitazoxanide stimulates autophagy and inhibits mTORC1 signaling and intracellular proliferation of Mycobacterium tuberculosis. PLoS Pathog 8: e1002691, 2012.

86. Tung SM, Unal C, Ley A, Pena C, Tunggal B, Noegel AA, Krut O, Steinert M and Eichinger L: Loss of Dictyostelium ATG9 results in a pleiotropic phenotype affecting growth, development, phagocytosis and clearance and replication of Legionella pneumophila. Cell Microbiol 12: 765-780, 2010.

87. Ye Y, Li X, Wang W, Ouedraogo KC, Li Y, Gan C, Tan S, Zhou X and Wu M: Atg7 deficiency impairs host defense against Klebsiella pneumoniae by impacting bacterial clearance, survival and inflammatory responses in mice. Am J Physiol Lung Cell Mol Physiol 307: L355-L363, 2014. 
88. Gannagé M, Dormann D, Albrecht R, Dengjel J, Torossi T, Rämer PC, Lee M, Strowig T, Arrey F, Conenello G, et al: Matrix protein 2 of influenza A virus blocks autophagosome fusion with lysosomes. Cell Host Microbe 6: 367-380, 2009.

89. Lee SJ, Smith A, Guo L, Alastalo TP, Li M, Sawada H, Liu X, Chen ZH, Ifedigbo E, Jin Y, et al: Autophagic protein LC3B confers resistance against hypoxia-induced pulmonary hypertension. Am J Respir Crit Care Med 183: 649-658, 2011.

90. Patel AS, Lin L, Geyer A, Haspel JA, An CH, Cao J, Rosas IO and Morse D: Autophagy in idiopathic pulmonary fibrosis. PLoS One 7: e41394, 2012.

91. Chen ZH, Kim HP, Sciurba FC, Lee SJ, Feghali-Bostwick C, Stolz DB, Dhir R, Landreneau RJ, Schuchert MJ, Yousem SA, et al: Egr-1 regulates autophagy in cigarette smoke-induced chronic obstructive pulmonary disease. PLoS One 3: e3316, 2008.
92. Chen ZH, Lam HC, Jin Y, Kim HP, Cao J, Lee SJ, Ifedigbo E, Parameswaran H, Ryter SW and Choi AM: Autophagy protein microtubule-associated protein 1 light chain-3B (LC3B) activates extrinsic apoptosis during cigarette smoke-induced emphysema. Proc Natl Acad Sci USA 107: 18880-18885, 2010.

93. Yang ZJ, Chee CE, Huang S and Sinicrope FA: The role of autophagy in cancer: Therapeutic implications. Mol Cancer Ther 10: 1533-1541, 2011

94. Kim EJ, Jeong JH, Bae S, Kang S, Kim CH and Lim YB: mTOR inhibitors radiosensitize PTEN-deficient non-small-cell lung cancer cells harboring an EGFR activating mutation by inducing autophagy. J Cell Biochem 114: 1248-1256, 2013.

95. Hsieh CH, Pai PY, Hsueh HW, Yuan SS and Hsieh YC: Complete induction of autophagy is essential for cardioprotection in sepsis. Ann Surg 253: 1190-1200, 2011. 